\title{
PROSODY STUDY WITH CONTEXT-DEPENDENT ACOUSTIC MODELS
}

\author{
Yue-Ning $\mathrm{Hu}^{1 *}, \mathrm{Min} C h u^{2}$ \\ ${ }^{1}$ School of Computer Science, Northwestern Polytechnical University, Xi'an, 710072 \\ ${ }^{2}$ Microsoft Research Asia, Beijing, 100080 \\ ynhu.moon@gmail.com, minchu@micrososft.com
}

\begin{abstract}
In this paper, we propose to study prosody with context-dependent acoustic models. We find that we can achieve better resolution on a specific aspect by training $\mathrm{CDM}$ with certain focus. For the tone recognition task, CDM with focus on tones should be used and it achieves $15.2 \%$ relative error reduction, when comparing with the traditional tri-phone models. For detecting prosody boundaries, CDM with focus on position should be used and the accuracy of prosodic word is $92.2 \%$. CDMs are also used to visualize the f0 patterns of sentences with give contextual information. Such patterns are helpful to understand the interaction among contextual factors. Overall, CDMs are useful data source for various prosody studies.
\end{abstract}

Index Terms - context-dependent model, model focus, prosody study, tone recognition, phrase boundary detection

\section{INTRODUCTION}

When Mandarin prosody is studied, it is important to understand the interactions between tone and intonation, between succeeding tones and the impact from other factors. The traditional way of prosody analysis is to design a phonetic experiment to study a specific topic. Many works have been done with such a method. Wu [1] [2] reported the tone variations in bi-syllabic words and tri-syllabic words. Shih [3] studied Mandarin tone sanhdi. Xu [4] studied the tone variations in different tonal context. However, limited by capability of collecting laboratory speech, it is difficult to study the interactions among multiple factors with such a designed experiment. Furthermore, the variations in natural speech cannot be fully captured by the laboratory speech. In this paper, we propose to study the prosody variations with context dependent acoustic models that are trained from a large scale speech corpus.

Context-dependent model (CDM) is not a new concept. A typical example of CDM is the tri-phone model, which has been widely used in the speech recognition task. In this case, only the phones before and after the current phone are considered as the factors that may cause segmental variations of the current phone. However, when prosody variation is to be studied, more factors should be considered. For Mandarin, the syllable sequence, the tone sequence and the prosodic structure of a sentence all play important roles in forming the sentence prosody. Besides, emotion and intention can make differences in prosody.

In this paper, we will focus on three aspects: phone, tone and prosodic structure. CDMs are constructed for the initial and final portion of a syllable, respectively. Each model is described by a multi- dimensional contextual vector, which includes 7 dimensions as shown in Table 1. Dimension 1-3 are about phones, i.e. current phone and the phone before and after it. Dimension 4-6 are about tones, i.e. the tone of the current syllable, the tone before and after the current syllables. The last one is about the prosodic structure. Two levels of prosodic structure, prosodic word and prosodic phrase, are considered. Each syllable has a position field to reflect its position within its carrying word and phrase. A syllable can have four possible positions in each level: initial, middle, final and standing alone as a word or a phrase (denoted as mono). Thus, there are total 16 possible positions. However, not all combinations are valid. For example, a syllable at phrase final could only be a word final or a mono word rather than a word middle or initial. We encode the syllable position with a 6-level schema: phrase initial (denoted as SP1) is the first syllable of a phrase; word initial (denoted as SP2) is the first syllable of a prosodic word, but not at phrase initial; SP3 denotes the syllable in the middle part of a prosodic word, and of course these syllables are in the middle part of a phrase; SP4 and SP5 stand for the word final and phrase final, respectively, and the former one is the last syllable of a prosodic word, but not a phrase final while the latter is the last syllable of a phrase; SP6 marks the mono-syllabic word that appears at any position within a phrase.

Table 1. Definition of the seven-dimensional contextual vector

\begin{tabular}{|c|l|}
\hline $\mathrm{LPh}$ & left phone, preceding phone of current phone \\
\hline $\mathrm{CPh}$ & current phone, initial or final of focused syllable \\
\hline $\mathrm{RPh}$ & right phone, succeeding phone of current phone \\
\hline $\mathrm{LT}$ & left tone, tones of the preceding syllable \\
\hline $\mathrm{CT}$ & current tone, tones of the focused syllable \\
\hline $\mathrm{RT}$ & right tone, tones of the succeeding syllable \\
\hline $\mathrm{SP}$ & syllable position, position label of current phone \\
\hline
\end{tabular}

More factors, such stress level, being semantic focus or not, emotional status, can be added to the contextual vector. We haven't used them in this paper because we don't have a train set containing such information.

This paper is organized as follows: CDM with focus are introduced in Section 2; In Section 3, the performance of CDM with different focus is compared in tone recognition task and prosodic boundary detection task. CDMs are also used to rank the impact of contextual factors and explore the tone variation in different context. The final conclusions are given in Section 4.

\section{CDM WITH FOCUS}

In order to have a statistical report for all contextual vectors, each of them is modeled by a multi-space distribution hidden Markov model

* The first author did the research as an intern in Microsoft Research Asia. 
(MSD-HMM) since it captures both continuous and discrete aspects of the fundamental frequency of speech well [5] [6]. Parameters of models are trained from a large scale speech corpus. Since the number of distinct vectors is very huge and is almost impossible to be fully covered by the training corpus, CART based state clustering is performed to group contextual vectors that have similar acoustic features together. We found that it is important to perform the state tying with different focus when solving different problems. For example, if the models are to be used for the tone recognition task, we would like to achieve the best tone discriminability. Then, states should be tied by tones, i.e. one tree per tone, to avoid any states sharing across tones. If the models are to be used for speech recognition, it is important to avoid states sharing across phones. Therefore, states are tied by phones. If the models are to be used to detect the phrase boundary from speech, states should be tied by syllable positions, i.e. one tree per position. Models with focus on current phones are referred as context dependent phone model (CDPhM). Tri-phone model belongs to this category. Similarly, models with focus on current tones or positions are named as context dependent tone model (CDTM) or context dependent position model (CDPM), respectively.

Details of training CDMs can be found in [6] [7]. In the next section, these models are compared in different tasks.

\section{PROSODY STUDY WITH CDM}

One of the main characters of CDM is that the focus could be changed. By changing focus, CDMs could be specified and used in different tasks of prosodic analysis.

\subsection{Speech corpus and setups}

A large Mandarin speech corpus read by a broadcast woman is used in our experiments. It is designed for speech synthesis and covers rich phonetic and prosodic variations. Prosodic structure [8] has been labeled manually, from which syllable position labels are derived automatically. The corpus contains 14476 utterances, 13476 of which are used for training and the remaining 1000 for testing. Some parameters are tuned with the first 100 utterances in the testing set.

Both segmental features (including 13-dimensional MFCC, 13-D $\Delta$ MFCC and 13-D $\triangle \Delta$ MFCC) and supra-segmental features (including logarithmic f0, its first and second order derivatives, pitch duration and long-span pitch [9]) are used.

\subsection{Tone recognition with CDTM}

Since we believe CDTM could capture tone variance more accurately than the other two types of models, it is used to recognize tones. We'd like to take advantage of the exiting HTK toolkits. CDTMs are not used to decode the speech directly. Instead, they are implemented to rescore the speech with known phone boundaries.

\subsubsection{Tone discriminability of CDM with different focus}

In order to verify the suitableness of using CDTM for the task, performance of CDTM is first compared with those of CDPhM and CDPM. The three kinds of CDMs are trained from the same speech corpus and the total numbers of parameters are constrained to the same level. To simplify the problem, we perform forced alignment with CDM candidates derived from the transcription of testing set on the testing set to get the phone boundaries. Then, we assume all phone information is known (i.e. $\mathrm{CPh}, \mathrm{LPh}$ and $\mathrm{RPh}$ are derived from transcription), but tone and position information are unknown. CDMs candidates in all possible contexts are then enumerated and the likelihood for each model to generate the corresponding acoustic observation is calculated. Finally, viterbi search with constraints is implemented to get the best tone path. These constraints aim to avoid the irregular tone or position sequence (see in [6] [7]). With such constraints, only reasonable tone sequence is to be found. The tone reorganization results for the three model sets are given in Table 2 . It is seen that CDTM significantly outperforms the other two model sets. This indicates that CDTMs have better discriminability on tones.

Table 2. Tone rescoring result of different CDMs

\begin{tabular}{|c|c|c|c|}
\hline CDMs & CDPhM & CDTM & CDPM \\
\hline Tone Accuracy (\%) & 90.24 & 93.04 & 89.57 \\
\hline
\end{tabular}

\subsubsection{Tone rescoring with CDTM}

In this experiment, the transcription of testing set is not used. Instead, we train traditional tri-phone models with the same training set and use them to decode the testing set. The error rate (ERR) of base syllables (syllables without considering tones) is only $3.8 \%$, yet, the tonally syllable ERR and tone ERR are $11.7 \%$ and $8.7 \%$, respectively. This shows that most errors are caused by tone confusions. The phone sequence and phone boundaries obtained by tri-phone decoding are then used in CDTM rescoring. In the same way as introduced in section 3.2.1, the best tone path could be achieved. The tone accuracy of CDTM is $92.6 \%$, comparing with the accuracy of tri-phone models $(91.3 \%), 15.2 \%$ relative error reduction is achieved. The result shows that CDTM has better tone discriminability than tri-phone models.

Table 3. Experiment result and tone confusion matrix

\begin{tabular}{|c|c|c|c|c|c|c|c|c|c|c|}
\hline \multirow{2}{*}{ rec } & \multicolumn{2}{|c|}{1} & \multicolumn{2}{c|}{2} & \multicolumn{2}{c|}{3} & \multicolumn{2}{c|}{4} & \multicolumn{2}{c|}{5} \\
\cline { 2 - 11 } & 4062 & \multicolumn{2}{c|}{5035} & \multicolumn{2}{c|}{3009} & \multicolumn{2}{c|}{6739} & \multicolumn{2}{c|}{1208} \\
\hline 1 & $\mathrm{~F}$ & $\mathrm{~S}$ & $\mathrm{~F}$ & $\mathrm{~S}$ & $\mathrm{~F}$ & $\mathrm{~S}$ & $\mathrm{~F}$ & $\mathrm{~S}$ & $\mathrm{~F}$ & $\mathrm{~S}$ \\
\hline 2 & 95.9 & 95.8 & 2.4 & 1.9 & 0.5 & 0.6 & 1.8 & 1.8 & 0.8 & 1.0 \\
\hline 3 & 2.0 & 1.5 & 94.2 & 94.0 & 7.0 & 5.1 & 0.8 & 0.5 & 2.2 & 1.7 \\
\hline 4 & 0.3 & 0.3 & 1.4 & 2.3 & 80.9 & 85.0 & 3.6 & 1.9 & 4.6 & 2.7 \\
\hline 5 & 0.1 & 2.4 & 1.2 & 1.5 & 9.3 & 8.8 & 92.7 & 95.1 & 10.0 & 13.1 \\
\hline
\end{tabular}

Note: F, the first-pass result, represents decode result of tri-phone model; S, second-pass result, stands for rescoring result of CDTM. Tone 5 denotes the neutral tone.

The tone confusion matrix for the two model sets are compared in Table 3. From Table 3, we find that CDTMs haven't shown benefits for tone 1 and 2, yet, it performs much better for tone 3 and tone 4 . The relative error reductions for the two tones are $21.5 \%$ and $33.3 \%$, respectively. One explanation for this is that tone 3 and tone 4 are affected more by their context. For example, a tone 4 at phrase initial often has much higher pitch level than a tone 4 at the phrase end. The accuracy of the neutral tone is slightly lower in the result of CDTM, which is caused by the higher confusion rate between tone 5 and tone 4 . One cause for this is the vague labeling criteria for neutral tone. We find that when a syllable with tone 4 appears at the end of a phrase and it is not stressed, the syllable is often neutralized. In some cases, it is labeled as tone 4 , and in others, as tone 5. Thus, the contextual tone model cannot distinguish the two tones in such situations.

Since the phone boundaries are got from the decoding results that contain deletion and insertion errors, the result of CDTM in this section is slightly worse than that in Table 2. CDTM has also been successfully applied in tone error detection in [10]. 


\subsection{Prosody boundary detection with CDPM}

Labeling prosodic boundaries in speech corpus is very useful for many prosody studies and speech synthesis. In the previous works, boundaries are either generated from the text transcription without looking at the real speech or labeled manually or semi-manually by listening to the speech. In this paper, we use CDPM to generate boundary labels automatically. Here, the task of boundary detection is converted to the task of syllable position labeling. In this case, the transcription of the test data is known and all the contextual factors except SP can be derived from the transcription. Phone boundaries are first obtained by forced alignment, and then CDM candidates with different SP are enumerated for all phones. Finally the best position path is obtained by Viterbi search with constraints on neighboring positions.

\subsubsection{Position discriminability of CDM with different focus}

Although, we believe that CDPM is most suitable for this task, the performances of the three CDM model sets are compared.

Though the boundary detection task has been converted to a syllable position labeling task, the position labels are converted back to word and phrase boundary marks with the following rules: any occurrence of a word final (SP4), phrase final (SP5) or a word mono (SP6) imply a word boundary after that syllable; whenever a SP5 or SP6 is followed by a SP1, or a SP5 followed by a SP6, a phrase boundary is marked. The performance of boundary detection is measured by precision and recall. For prosodic word boundary, the overall accuracy is given as well. The results are shown in Table 4.

Table 4. Prosody boundary detection result with CDM

\begin{tabular}{|l|c|c|c|c|c|}
\hline \multirow{2}{*}{$\begin{array}{l}\text { Model } \\
\text { sets }\end{array}$} & \multicolumn{2}{|c|}{ Prosodic Phrase (\%) } & \multicolumn{3}{|c|}{ Prosodic Word (\%) } \\
\cline { 2 - 6 } & recall & precision & recall & Precision & accuracy \\
\hline CDPM & 80.46 & 83.75 & 90.15 & 96.14 & 92.17 \\
\hline CDTM & 77.67 & 79.23 & 80.79 & 94.22 & 87.48 \\
\hline CDPhM & 76.42 & 72.58 & 83.13 & 91.52 & 86.20 \\
\hline
\end{tabular}

It is seen that CDPM outperforms others as expected. This confirms our assumption that CDPMs capture the acoustic characteristic of prosodic boundary better than the other two models. And it achieves rather good accuracy, especially for prosodic word.

\subsubsection{Error analysis}

In order to have a better understand of the capability of CDPM, we look into the errors in boundary detection. The confusion matrix of wordlevel position is given in Table 5 .

The confusion matrix shows that the confusion between word head and mid is significant, so does the confusion between word tail and mid. Besides, word mono is easily treated as word head or word tail. However, word head and word tail are seldom confused.

We have done more detailed analysis of these errors and design a listening experiment to verify whether parts of these errors were caused by randomness in manually labeling, i.e. some not very significant boundaries are labeled in some cases and not in other cases. Details of the experiment are described in [7]. The result shows that some errors are not real errors. In fact, only $28 \%$ of word boundaries errors and $50 \%$ of the phrase boundary errors are judged as worse than the manual labels. Since the error rate in word boundaries is $7.8 \%$ and only about $28 \%$ of them are real errors, the chance of getting a wrong word boundary is only $2.2 \%$. This is very close to the human labeling error rate reported in [11]. This result double confirms the capability of the CDPM in capture the characteristics of word boundaries.

Table 5. Confusion matrix of SP in prosodic words

\begin{tabular}{|l|c|c|c|c|}
\hline red \% & head & mid & tail & mono \\
\hline head & 8477 & 2167 & 8477 & 932 \\
\hline mid & 86.91 & 11.58 & 1.96 & 21.89 \\
\hline tail & 8.64 & 81.82 & 7.99 & 0.32 \\
\hline mono & 1.90 & 6.60 & 88.83 & 9.98 \\
\hline
\end{tabular}

Note: one head error will cause a tail error, thus the two errors are recorded in this table for each boundary error. So the word-level accuracy is higher than the four accuracies separately.

\subsection{Prosody analysis with CDM}

Besides tone recognition and prosody boundary detection, CDM could also be applied in analysis of speech data including studying the importance of contextual factors, generating the visualized fo pattern of the sentence.

\subsubsection{Ranking the importance of contextual factors}

Although 7 contextual factors are considered, the importance of each factor is not the same, and it is reflected to a great extent by the splitting questions of the decision trees for tying states. The earlier a question is used, the more important it is. Therefore, a factor is weighted by sum of the reciprocal of the depth of all questions regarding to it (see in [6]). Take CDTM as an example. The importance of each factor on each state is plotted in Figure 1.

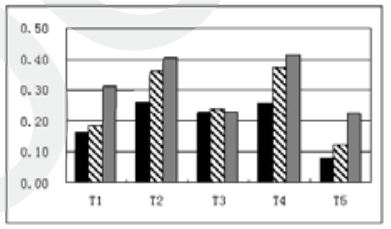

(a) SP

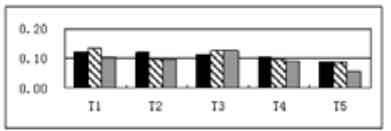

(c) $\mathrm{CP}$

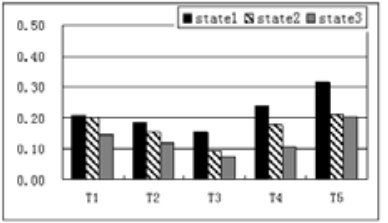

(b) LT

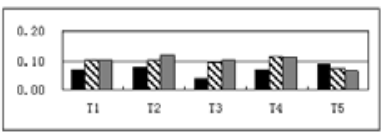

(d) RT
Figure 1. The importance of contextual factors (by tones and by states)

Among the six contextual factors we explore, SP is the most important one and LT ranks the second. Both $\mathrm{RT}$ and $\mathrm{CPh}$ influence the pitch level of tones, but their impact is weaker. The impact of LPh and $\mathrm{RPh}$ is the weakest, so we haven't shown them here. We further find that the impact of a factor varies across tones as well as the three states of the same tone. Normally, onset pitch of a tone is determined jointly by SP and LT, while, the offset pitch is mainly affected by SP, except for the neutral tone. All the three states of the neutral tone are mainly affected by LT and its last state is jointly affected by SP, More analysis impact from different factors and their interactions can be found in [6]. All these discoveries are consistent with those from previous studies and we can tell more about the interactions among factors. 


\subsubsection{Generating stylized $f_{0}$ pattern with CDPM}

A CDM represents the fo pattern in the given context. To better study the influence of syllable position on tones, we use CDPMs to produce the stylized fo pattern of a given phrase. We design two sets of phrases that have the same prosodic structure. They all contain a three-syllabic word, a mono-syllabic word and a bi-syllabic word. These phrases have fixed base syllables and have different tones for some syllables. The first group of phrases start with a tone 1 syllable and the second group start with a tone 4 syllable. To visualize the tone variations in different context, the tone of the second and fifth syllables in the two sets of phrases are enumerated from tone 1 to tone 4, as shown in Figure 2. The six states of one syllable represent the onset, main and offset state of the initial and the final portion, respectively. We use the darkness of the points to show the probability of being voiced of a state. The three states for the initial part often have grey points, and they have white points in some cases.

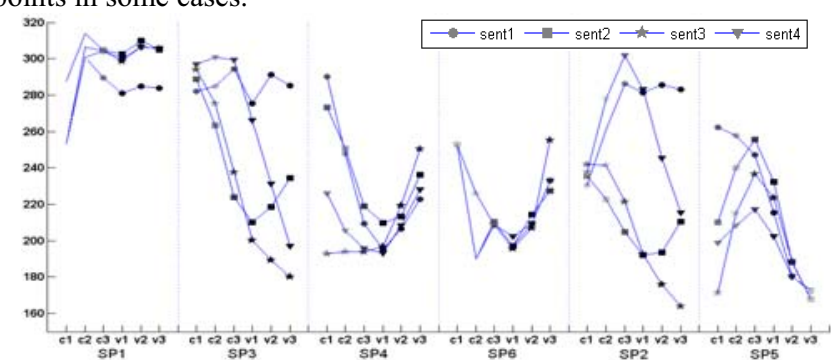

(a)

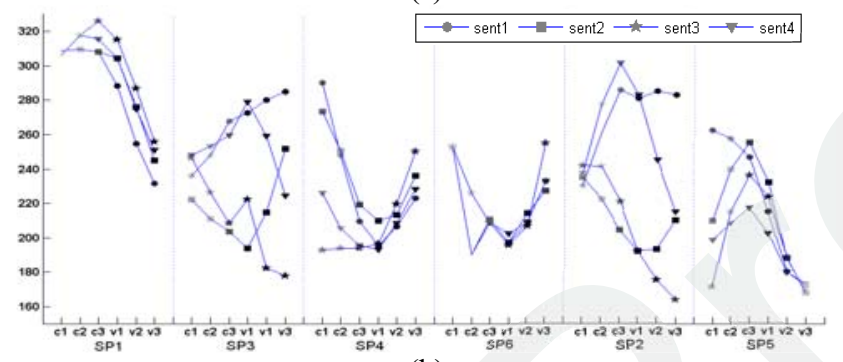

(b)

Figure 2. Stylized f0 pattern of two Chinese sentences

Note: Sent in (a): 张星(行, 醒, 幸)辰和马(妈, 麻, 骂)丽|zhang1 xing1(xing2, xing3, xing4) chen2 \# he2 \# ma3(ma1, ma2, ma4) li4; Sent in (b): 赵星(行, 醒, 幸)辰和马(妈, 麻, 骂)丽 $\mid$ zhao4 xing1(xing2, xing3, xing4) chen2 \# he2 \# ma3(ma1, ma2, ma4) li4; To compare the difference, the tones of second syllable (xing) and fifth syllable (ma) in the two sentences are changed from tone 1 to tone 4 , thus 4 sentences are got separately: sent1 4; \# marks the prosodic word boundaries, number 1-4 mark the four Mandarin tones and 5 represents the neutral tone.

Here are some observations: (1) all the four tones at SP3 (the second syllable) tend to start higher when preceded by a high-ending tone, compared to the case when it is preceded by a low-ending tone. The transition part mainly happens in the syllable initial part. If we watch the final part only, the observation is the reduction of pitch range after a low-ending tone. (2) The syllable at SP4 (the third syllable) has tone 2 and its starting pitch level is also affected by the preceding tone. Tone 1 and tone 2 tend to end with high pitch, and the following tone will start from high pitch. Tone 3 and tone 4 tend to end with low pitch and will cause the following tone start from low pitch. There are many other phenomena can be seen from the two figures. Due to the limitation of space, we will not list all. Here, we want to point out that such contextdependent models embed lots of useful information and can be a very good data source for various prosodic studies.

\section{CONCLUSIONS}

In this paper, we propose to use context-dependent acoustic models as a data source for various prosody studies. Each speech segment can be described by a multi-dimensional contextual vector. Each vector is then modeled by a MSD-HMM and can be trained from a speech corpus with different focus. CDTMs have better discriminative in tones and CDPMs are better for distinguishing syllable positions. In the tone recognition task, CDTM achieves $15.2 \%$ relative error reduction, when compared with the traditional tri-phone models; in the task of prosody boundary detection with CDPM, the real error rate for word boundaries is only about $2.2 \%$, which is very close to the human labeling error rate reported in [11]; CDMs are also used to visualize the f0 patterns of the given sentence patterns for better understanding of the interaction among contextual factors. Overall, CDMs are useful data source for various prosody studies. Properly adjusting the model focus can help achieve better resolution on a specific aspect.

In this paper, all experiments are done with a single speaker speech corpus. In the next step, we will test the approach with a multi-speaker corpus.

\section{REFERENCES}

[1] Wu Zongji, "Pu tong hua yu ju zhong de sheng diao bian hua", Zhongguo Yuwen, vol6. 1982, p439-449. (In Chinese)

[2] Wu Zongji, "Pu tong hua san zi zu bian diao gui lv", Journal of Chinese Linguistics, vol2, 1985, p70-92. (In Chinese)

[3] Chilin Shih, "Mandarin Third Tone Sandhi and Prosodic Structure", In Studies in Chinese Phonology, J. Wang and N. Smith, Mouton de Gruyter, p81-123.

[4] Yi Xu, "Contextual tonal variations in Mandarin", Journal of Phonetics, 25, 61-83.

[5] Tokuda K., Masuko T., et al., "Hidden markov models based on multi-space probability distribution for pitch pattern modeling", Proc. of ICASSP, 1999.

[6] Yue-Ning Hu, Min Chu, Chao Huang, et al., "Exploring tonal variations via context-dependent tone models", Proc. of Interspeech, 2007.

[7] Yue-Ning Hu, Min Chu, Chao Huang, et al., "Prosody boundary detection through context-dependent position models", Proc. of Interspeech, 2008.

[8] Chu, M., Peng, H., Yang, H. Y. and Chang, E., "Selecting Nonuniform Units from a Very Large Corpus for Concatenative Speech Synthesizer", Proc. of ICASSP, 2001.

[9] Zhou J., Tian Y., et al., "Tone articulation modeling for mandarin spontaneous speech recognition", Proc. of ICASSP, 2004.

[10] Yan-Bin Zhang, Min Chu, et al., "Detecting tone errors in continuous mandarin speech", Proc.of ICASSP, 2007.

[11] Lin $\mathrm{He}$, Min Chu, et al., "A study on labeling prosody hierarchy in Mandarin speech corpus", The 5th National Conference on Modern Phonetics, 2001. (In Chinese) 